\title{
An innovative linear complexity computation for cryptographic sequences *
}

\author{
J.L. Martín-Navarro ${ }^{1}$, Amparo Fúster-Sabater ${ }^{1}$ and Sara D. Cardell ${ }^{2}$ \\ 1 Instituto de Tecnologías Físicas y de la Información, C.S.I.C. \\ Serrano 144, 28006 Madrid, Spain. \\ jomarna6@inf.upv.es amparo@iec.csic.es \\ 2 Instituto de Matemática, Estatística e Computação Científica \\ UNICAMP, R. Sérgio Buarque de Holanda, 651, \\ 13083-859, Campinas-SP, Brazil \\ sdcardell@ime.unicamp.br
}

\begin{abstract}
A simple algorithm to compute the linear complexity of binary sequences with period a power of 2 has been proposed. The algorithm exploits the fractal structure of the binomial representation in this kind of binary sequences. The application of the general algorithm to a particular family of cryptographic sequences (generalized sequences) improves its performance as decreases the amount of sequence to be processed.
\end{abstract}

Keywords: binomial sequence $\cdot$ generalized generator $\cdot$ linear complexity · Sierpinski triangle.

\section{Introduction}

Nowadays, Internet of Things (IoT) is one of the hot topics in computer science and information technologies. In the near future, IoT will be used more and more to connect many and different types of devices. Some of them use powerful processors that allow them to perform the same cryptographic algorithms as those of standard PCs. However, many others use extremely low power microcontrollers that can hardly devote a small fraction of their computing power to security. At any rate, cryptographic algorithms must be implemented in the communication among items in order to provide security, authenticity and integrity in the message exchange. Due to the very low energy available in many of the interconnected devices, the cryptographic algorithms have to be as light as possible. It is inside this kind of lightweight cryptography where stream ciphers, the simplest and fastest among all the encryption procedures, play a leading part.

* Research partially supported by Ministerio de Economía, Industria y Competitividad, Agencia Estatal de Investigación, and Fondo Europeo de Desarrollo Regional (FEDER, UE) under project COPCIS (TIN2017-84844-C2-1-R) and by Comunidad de Madrid (Spain) under project CYNAMON (P2018/TCS-4566), also co-funded by European Union FEDER funds. The first author was supported by JAE INTRO'19. 
The main concern in stream cipher design is to generate from a short and truly random key a long and pseudo-random sequence called keystream sequence to be used in the encryption/decryption procedure. Long period, large linear complexity or good statistical properties are some of the requirements that every keystream sequence must satisfy. Linear complexity is a largely used metric to guarantee security in a cryptographic sequence. Roughly speaking, linear complexity is a measure of the amount of sequence we have to know in order to recover the whole sequence.

In this work, an efficient algorithm to compute the linear complexity of binary sequences with period $T=2^{m}, m$ being an integer, has been developed. The algorithm is based on the binomial representation of binary sequences. Indeed, a binary sequence can be expressed as a linear combination of binomial sequences that allow one to analyse the cryptographic parameters of such a sequence. Moreover, the algorithm here proposed exploits the fractal structure of the binomial sequences and reduces the computation of the linear complexity to $m$ bit-wise additions of the sequence bits.

Although the algorithm is general and can be applied to every binary sequence with $T=2^{m}$, in practice we focus on a family of cryptographic sequences, the generalized sequences (see section 2), patented in [13] for cryptographic purposes. In this case, the efficiency of the algorithm increases as the amount of sequence required decreases.

This paper is organized as follows. Section 2 includes a succinct revision of the linear feedback shift registers and the family of generalized sequences. In Section 3 , the binomial representation of binary sequences is addressed as well as the characteristics and properties of the binomial sequences are studied. Afterwards, Section 4 develops an algorithm for computing the linear complexity of binary sequences as well as its particularization to the generalized sequences. Finally, conclusions and possible future research lines end the work.

\section{LFSR-based sequence generators: the generalized self-shrinking generator}

Linear Feedback Shift Registers (LFSRs) [9] are linear structures currently used in the generation of pseudorandom sequences. The LFSRs are electronic devices with $L$ memory cells or stages. They are included in most of the sequence generators proposed in the literature. The main reasons for a so generalized use are: LFSRs provide high performance when used for sequence generation, they are particularly well-suited to hardware implementations and, due to their linear structure, such registers can be readily analysed by means of algebraic techniques.

The LFSR generates an output sequence $\left\{a_{n}\right\}(n=0,1,2, \ldots)$ by means of shifts and linear feedbacks. If the connection polynomial is primitive [9], then the LFSR is a maximal-length LFSR and the output sequence is called PNsequence (pseudo-noise sequence). Such a PN-sequence has period $T=2^{L}-1$ bits with $2^{L-1}$ ones and $2^{L-1}-1$ zeros. Linear complexity $(L C)$ of a sequence 
is a parameter closely related to the concept of LFSR. In fact, $L C$ is defined as the length of the shortest LFSR able to generate such a sequence.

Although an LFSR in itself is an excellent generator of pseudo-random sequence, nevertheless it has undesirable linearity properties which reduce the security of its use. Even if the feedback polynomial is kept secret, the knowledge of $2 \cdot L$ output bits and the Berlekamp-Massey algorithm [11] can determine the whole sequence. In practice, the introduction of any type of non-linearity in the process of formation of the pseudo-random sequence is needed. The irregular decimation of $\mathrm{PN}$-sequences is one of the most popular techniques to destroy the inherent linearity of the LFSRs $[3,6]$.

Among the types of irregularly decimated generators, we can enumerate: 1) the shrinking generator introduced in [5] that includes two LFSRs, 2) the selfshrinking generator [12] based on self-decimation with just one LFSR and 3) the generalized self-shrinking generator proposed in [10] that produces a family of cryptographic sequences. In this work, we focus on the generalized self-shrinking generator patented in [13].

\subsection{The generalized self-shrinking generator}

The generalized self-shrinking generator can be described as follows:

1. It makes use of two PN-sequences: $\left\{a_{n}\right\}$ a PN-sequence produced by a maximal-length LFSR with $L$ stages and a shifted version of such a sequence denoted by $\left\{v_{n}\right\}$. In fact, $\left\{v_{n}\right\}=\left\{a_{n+p}\right\}$ corresponds to the own sequence $\left\{a_{n}\right\}$ but rotated cyclically $p$ positions to the left with $\left(p=0,1, \ldots, 2^{L}-2\right)$.

2. It relates both sequences by means of a simple decimation rule to generate the output sequence. For $n \geq 0$, we define the decimation rule as follows:

$$
\left\{\begin{array}{l}
\text { If } a_{n}=1 \text { then } v_{n} \text { is output, } \\
\text { If } a_{n}=0 \text { then } v_{n} \text { is discarded and there is no output bit. }
\end{array}\right.
$$

Thus, for each value of $p$ an output sequence $\left\{s_{n}\right\}_{p}=\left\{s_{0} s_{1} s_{2} \ldots\right\}_{p}$ is generated. Such a sequence is called the $p$ generalized self-shrunken sequence or simply generalized sequence associated with the shift $p$. Recall that $\left\{a_{n}\right\}$ remains fixed while $\left\{v_{n}\right\}$ is the sliding sequence or left-shifted version of $\left\{a_{n}\right\}$. When $p$ ranges in the interval $\left[0,1, \ldots, 2^{L}-2\right]$, then we obtain the $2^{L}-1$ members of the family of generalized self-shrunken sequences based on the PN-sequence $\left\{a_{n}\right\}$. Since the PN-sequence has $2^{L-1}$ ones, the period of any generalized sequence will be $2^{L-1}$ or divisors, that is a power of 2 . Moreover, in $[2,8]$ an upper-bound on the $L C$ of generalized sequences is provided, that is

$$
L C \leq 2^{L-1}-(L-2) .
$$

Let us see a simple example. For an LFSR with primitive polynomial $p(x)=$ $x^{4}+x+1$ and initial state $\left(\begin{array}{llll}1 & 1 & 1 & 1\end{array}\right)$, we generate the generalized sequences depicted in Table 1 . The bits in bold in the different sequences $\left\{v_{n}\right\}$ are the digits of the corresponding generalized sequences associated to their corresponding shifts $p$ plus the identically null sequence. The PN-sequence $\left\{a_{n}\right\}$ with period $T=2^{4}-1$ is written at the bottom of the table in columns 2 and 5 . 
Table 1: GSS-sequences for an LFSR with polynomial $p(x)=x^{4}+x+1$

\begin{tabular}{|c|c|c||c|c|c|}
\hline shift $p$ & $\left\{v_{n}\right\}$ sequences & GSS-sequences & shift $p$ & $\left\{v_{n}\right\}$ sequences & GSS-sequences \\
\hline 0 & $\mathbf{1 1 1 1 0 0 0 1 0 0 1 1 0 1 0}$ & 11111111 & 8 & $\mathbf{0 0 1 1 0 1 0 1 1 1 \mathbf { 0 } 0 0 1}$ & 00111100 \\
1 & $\mathbf{1 1 1 0 0 0 1 0 0 1 1 0 1 0 1}$ & 11100100 & 9 & $\mathbf{0 1 1 0 1 0 1 1 1 1 0 0 0 1 0}$ & 01101001 \\
2 & $\mathbf{1 1 0 0 0 1 0 0 1 1 0 1 0 1 1}$ & 11000011 & 10 & $\mathbf{1 1 0 1 0 1 1 1 1 0 0 0 1 0 0}$ & 11011000 \\
3 & $\mathbf{1 0 0 0 1 0 0 1 1 0 1 0 1 1 1}$ & 10001101 & 11 & $\mathbf{1 0 1 0 1 1 1 1 0 0 0 1 0 0 1}$ & 10101010 \\
4 & $\mathbf{0 0 0 1 0 0 1 1 0 1 0 1 1 1 1}$ & 00011011 & 12 & $\mathbf{0 1 0 1 1 1 1 0 0 0 1 0 0 1 1}$ & 01010101 \\
5 & $\mathbf{0 0 1 0 0 1 1 0 1 0 1 1 1 1 0}$ & 00100111 & 13 & $\mathbf{1 0 1 1 1 1 0 0 0 1 0 0 1 1 0}$ & 10110001 \\
6 & $\mathbf{0 1 0 0 1 1 0 1 0 1 1 1 1 0 0}$ & 01001110 & 14 & $\mathbf{0 1 1 1 1 0 0 0 1 0 0 1 1 0 1}$ & 01110010 \\
7 & $\mathbf{1 0 0 1 1 0 1 0 1 1 1 1 0 0 0}$ & 10010110 & - & $\mathbf{0 0 0 0 0 0 0 0 0 0 0 0 0 0 0}$ & 00000000 \\
\hline \hline
\end{tabular}

\section{Binomial sequences, Sierpinski's triangle and cellular automata}

In this section, we introduce a new representation of binary sequences whose period is a power of 2 in terms of the binomial sequences. Next, the close relationship among binomial sequences, the Sierpinski's triangle and a type of linear cellular automata is also analysed.

\subsection{Binomial sequences}

The binomial number $\left(\begin{array}{c}n \\ i\end{array}\right)$ is the coefficient of the power $x^{i}$ in the polynomial expansion of $(1+x)^{n}$. For every positive integer $n$, it is a well-known fact that $\left(\begin{array}{l}n \\ 0\end{array}\right)=1$ as well as $\left(\begin{array}{c}n \\ i\end{array}\right)=0$ for $i>n$. The binomial coefficients reduced modulo 2 allow us to define the concept of binomial sequence.

Definition 1. Given an integer $i \geq 0$, the sequence $\left\{b_{n}\right\}_{i}(n=0,1,2, \ldots)$ whose elements are binomial coefficients reduced modulo 2 , that is $b_{n}=\left(\begin{array}{c}n \\ i\end{array}\right) \bmod 2$, is called the $i$-th binomial sequence.

Table 2: The eight first binomial sequences, their periods and linear complexities

\begin{tabular}{|c|c|c|c|}
\hline Binomial coeff. & Binomial sequences $\left.\left\{\begin{array}{l}n \\
i\end{array}\right)\right\}$ & Period & Linear complexity \\
\hline$\left(\begin{array}{c}n \\
0\end{array}\right)$ & $\{1,1,1,1,1,1,1,1, \ldots\}$ & $T_{0}=1$ & $L C_{0}=1$ \\
$\left(\begin{array}{c}n \\
1\end{array}\right)$ & $\{0,1,0,1,0,1,0,1, \ldots\}$ & $T_{1}=2$ & $L C_{1}=2$ \\
$\left(\begin{array}{c}n \\
2\end{array}\right)$ & $\{0,0,1,1,0,0,1,1, \ldots\}$ & $T_{2}=4$ & $L C_{2}=3$ \\
$\left(\begin{array}{c}n \\
3\end{array}\right)$ & $\{0,0,0,1,0,0,0,1, \ldots\}$ & $T_{3}=4$ & $L C_{3}=4$ \\
$\left(\begin{array}{c}n \\
4\end{array}\right)$ & $\{0,0,0,0,1,1,1,1, \ldots\}$ & $T_{4}=8$ & $L C_{4}=5$ \\
$\left(\begin{array}{c}n \\
5\end{array}\right)$ & $\{0,0,0,0,0,1,0,1, \ldots\}$ & $T_{5}=8$ & $L C_{5}=6$ \\
$\left(\begin{array}{c}n \\
6\end{array}\right)$ & $\{0,0,0,0,0,0,1,1, \ldots\}$ & $T_{6}=8$ & $L C_{6}=7$ \\
$\left(\begin{array}{c}n \\
7\end{array}\right)$ & $\{0,0,0,0,0,0,0,1, \ldots\}$ & $T_{7}=8$ & $L C_{7}=8$ \\
\hline
\end{tabular}


In brief, a binomial sequence is a binary sequence whose terms are binomial numbers reduced mod 2. Table 2 shows the eight first binomial sequences $\left\{b_{n}\right\}_{i}=\left\{\left(\begin{array}{c}n \\ i\end{array}\right)\right\},(i=0,1, \ldots, 7)$, with their corresponding periods $T_{i}$ and linear complexities $L C_{i}$, see [7]. Next, different properties of the binomial sequences are enumerated:

1. Given the binomial sequence $\left\{\left(\begin{array}{c}n \\ 2^{L}+k\end{array}\right)\right\}$, with $0 \leq k<2^{L}$, then we have that [4, Proposition 1.b]:

a) Such a binomial sequence has period $T=2^{L+1}$.

b) The first period of such a binomial sequence has the following structure:

$$
\left\{\left(\begin{array}{c}
n \\
2^{L}+k
\end{array}\right)\right\}_{0 \leq n<2^{L+1}}=\left\{\begin{array}{lll}
0 & \text { if } \quad 0 \leq n<2^{L}+k \\
\left(\begin{array}{l}
n \\
k
\end{array}\right) \bmod 2 & \text { if } \quad 2^{L}+k \leq n<2^{L+1}
\end{array}\right.
$$

2. The linear complexity of the binomial sequence $\left\{\left(\begin{array}{c}n \\ i\end{array}\right)\right\}$ with $0 \leq i$ is $L C=$ $i+1$, see [4, Theorem 3].

3. Every binary sequence whose period is a power of 2 can be written as a linear combination of a finite number of binomial sequences [4, Theorem 1]. Such a combination is called the binomial representation of the sequence.

4. Given a sequence with binomial representation $\sum_{k=1}^{t}\left\{\left(\begin{array}{c}n \\ i_{k}\end{array}\right)\right\}$, where $i_{1}<$ $i_{2}<\cdots<i_{t}$ are integer indexes, then its linear complexity is given by $L C=i_{t}+1$, see [7].

5. Given a sequence with binomial representation $\sum_{k=1}^{t}\left\{\left(\begin{array}{l}n \\ i_{k}\end{array}\right)\right\}$, where $i_{1}<$ $i_{2}<\cdots<i_{t}$ are integer indexes, then its period $T$ is that of the binomial sequence $\left\{\left(\begin{array}{l}n \\ i_{t}\end{array}\right)\right\}$, see [7].

Recall that the generalized sequences are binary sequences whose period is a power of 2. Consequently, they can be written in terms of binomial sequences satisfying all the previous properties.

\subsection{Sierpinski's triangle and cellular automata}

When the binomial coefficients are arranged into rows for the successive values of $n=0,1,2, \ldots$, then the generated structure is the Pascal's triangle (see Figure 1a). If we color the odd numbers and shade the even ones in such a triangle, then we get the Sierpinski's triangle whose version reduced mod 2 is depicted in Figure 1b.

Recall that the successive diagonals of the Sierpinski's triangle in Figure 1b correspond to the successive binomial sequences $\left\{\left(\begin{array}{c}n \\ i\end{array}\right)\right\},(i=0,1,2, \ldots)$ starting at the first 1 . That is, the binomial sequences can be found inside the Sierpinski's triangle mod 2.

On the other hand, cellular automata (CA) are discrete structures composed of a finite number of cells whose content is updated according to a rule or function with $k$ variables [14]. The state of the cell in position $i$ at time $t+1$, notated 
Fig. 1

(a) Pascal's triangle

$\left(\begin{array}{l}0 \\ 0\end{array}\right)$

$\left(\begin{array}{ll}1 \\ 0\end{array}\right) \quad\left(\begin{array}{l}1 \\ 1\end{array}\right)$

$\left(\begin{array}{lll}2 \\ 0\end{array}\right) \quad\left(\begin{array}{l}2 \\ 1\end{array}\right) \quad\left(\begin{array}{l}2 \\ 2\end{array}\right)$

$\left(\begin{array}{llll}3 \\ 0\end{array}\right) \quad\left(\begin{array}{l}3 \\ 1\end{array}\right) \quad\left(\begin{array}{l}3 \\ 2\end{array}\right) \quad\left(\begin{array}{l}3 \\ 3\end{array}\right)$

$\left(\begin{array}{llll}4 \\ 0\end{array}\right) \quad\left(\begin{array}{l}4 \\ 1\end{array}\right) \quad\left(\begin{array}{l}4 \\ 2\end{array}\right) \quad\left(\begin{array}{l}4 \\ 3\end{array}\right) \quad\left(\begin{array}{l}4 \\ 4\end{array}\right)$

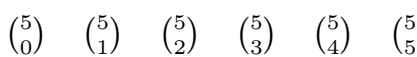

(b) Sierpinski's triangle mod 2

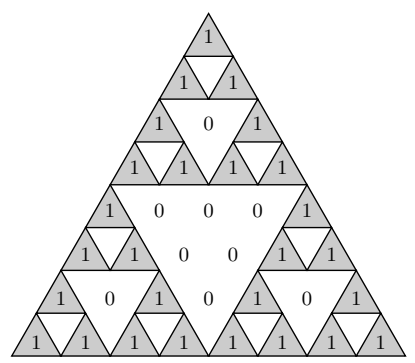

Fig. 2: CA-images generated with rules 102 and 60

Rule 102

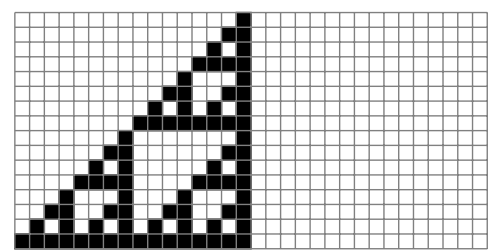

Rule 60

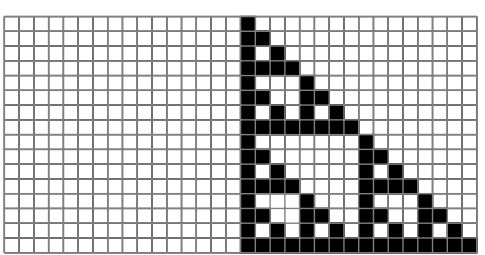

$x_{i}^{t+1}$, depends on the state of the $k$ neighbour cells at time $t$. If these rules are composed exclusively of XOR operations, then the CA are linear. The CA here considered are regular (every cell follows the same rule) and one-dimensional. For $k=3$, rules 102 and 60 are defined as follows:

$$
\text { Rule 102: } \quad x_{i}^{t+1}=x_{i}^{t}+x_{i+1}^{t} \quad \text { Rule 60: } \quad x_{i}^{t+1}=x_{i-1}^{t}+x_{i}^{t}
$$

In Figure 2, these rules are depicted according to Wolfram terminology [14]: a white square represents the digit 0 and a black square represents the digit 1 .

Recall that, in Figure 2, the successive columns of rule 102-image (numbered from right to left) correspond to the successive binomial sequences $\left\{\left(\begin{array}{l}n \\ i\end{array}\right)\right\}$, $(i=0,1,2, \ldots)$. The same applies for the successive columns of rule 60 -image (numbered from left to right). That is, the binomial sequences can be found inside certain one-dimensional linear regular CA [1].

In brief, the binomial sequences previously defined can be found in well known mathematical structures, e.g. Pascal's triangle, Sierpinski's triangle or certain types of cellular automata.

\section{An efficient algorithm for computing the LC}

An efficient algorithm that computes the linear complexity of binary sequences whose period is $T=2^{m}, m$ being an integer $m>0$, is now introduced. This 
Table 3: The 32 first binomial sequences

\begin{tabular}{|c|c|c|}
\hline 111111 & & \\
\hline 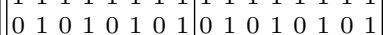 & 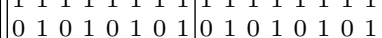 & \\
\hline 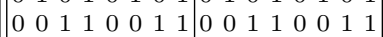 & $\begin{array}{lllllllllllllll}0 & 0 & 1 & 1 & 0 & 0 & 1 & 1\end{array} \mid \begin{array}{lllllllll}0 & 0 & 1 & 1 & 0 & 0 & 1 & 1\end{array}$ & \\
\hline \begin{tabular}{llllllll|lllllll}
0 & 0 & 0 & 1 & 0 & 0 & 0 & 1 & 0 & 0 & 0 & 1 & 0 & 0 & 0
\end{tabular} & \begin{tabular}{llllllll|llllllll}
0 & 0 & 0 & 1 & 0 & 0 & 0 & 1 & 0 & 0 & 0 & 1 & 0 & 0 & 0 & 1
\end{tabular} & \\
\hline $\mid$\begin{tabular}{llllll|llllllll}
0 & 0 & 1 & 1 & 1 & 1 & 0 & 0 & 0 & 0 & 1 & 1 & 1 & 1
\end{tabular} & 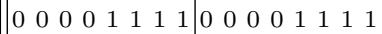 & \\
\hline \begin{tabular}{llllllll|llllllll}
0 & 0 & 0 & 0 & 0 & 1 & 0 & 1 & 0 & 0 & 0 & 0 & 0 & 1 & 0 & 1
\end{tabular} & $\left.\mid \begin{array}{llllllll|lllllll}0 & 0 & 0 & 0 & 0 & 1 & 0 & 1 & 0 & 0 & 0 & 0 & 0 & 1 & 0\end{array}\right]$ & \\
\hline \begin{tabular}{lllllll|llllllll}
0 & 0 & 0 & 0 & 0 & 0 & 1 & 1 & 0 & 0 & 0 & 0 & 0 & 0 & 1
\end{tabular} & & \\
\hline$\left[\begin{array}{llllllll|lllllll}0 & 0 & 0 & 0 & 0 & 0 & 0 & 1 & 0 & 0 & 0 & 0 & 0 & 0 & 0\end{array}\right]$ & {$\left[\begin{array}{lllllll|lllllll}0 & 0 & 0 & 0 & 0 & 0 & 0 & 1\end{array} \mid \begin{array}{lllllllll}0 & 0 & 0 & 0 & 0 & 0 & 1\end{array}\right]$} & \\
\hline \begin{tabular}{|llllllll|lllllll|} 
& 0 & 0 & 0 & 0 & 0 & 0 & 0 & 1 & 1 & 1 & 1 & 1 & 1 & 1
\end{tabular} & \begin{tabular}{|lllllllllllllllll}
0 & 0 & 0 & 0 & 0 & 0 & 0 & 0 & 1 & 1 & 1 & 1 & 1 & 1 & 1 & 1
\end{tabular} & \\
\hline 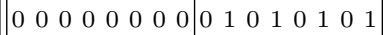 & $\mid$\begin{tabular}{llllllll|llllllll}
0 & 0 & 0 & 0 & 0 & 0 & 0 & 0 & 0 & 1 & 0 & 1 & 0 & 1 & 0 & 1
\end{tabular} & \\
\hline $\left.\begin{array}{llllllll|lllllll}0 & 0 & 0 & 0 & 0 & 0 & 0 & 0 & 0 & 0 & 1 & 1 & 0 & 0 & 1\end{array}\right]$ & $\mid \begin{array}{llllllllllllll}0 & 0 & 0 & 0 & 0 & 0 & 0 & 0 & 0 & 0 & 1 & 1 & 0 & 0\end{array}$ & \\
\hline$\left[\begin{array}{llllllll|lllllll}0 & 0 & 0 & 0 & 0 & 0 & 0 & 0 & 0 & 0 & 0 & 1 & 0 & 0 & 0\end{array}\right]$ & $\left.\mid \begin{array}{llllllll|lllllll}0 & 0 & 0 & 0 & 0 & 0 & 0 & 0 & 0 & 0 & 0 & 1 & 0 & 0 & 0\end{array}\right]$ & \\
\hline 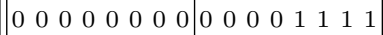 & $\left.\mid \begin{array}{llllllll|llllllll}0 & 0 & 0 & 0 & 0 & 0 & 0 & 0 & 0 & 0 & 0 & 0 & 1 & 1 & 1\end{array}\right]$ & \\
\hline$\left[\begin{array}{llllllll|lllllll}0 & 0 & 0 & 0 & 0 & 0 & 0 & 0 & 0 & 0 & 0 & 0 & 0 & 1 & 0\end{array}\right]$ & $\left.\mid \begin{array}{lllllllllllllll}0 & 0 & 0 & 0 & 0 & 0 & 0 & 0 & 0 & 0 & 0 & 0 & 0 & 1 & 0\end{array}\right]$ & \\
\hline$\left[\begin{array}{llllllll|lllllll}0 & 0 & 0 & 0 & 0 & 0 & 0 & 0 & 0 & 0 & 0 & 0 & 0 & 0 & 1\end{array}\right]$ & $\mid$\begin{tabular}{llllllll|llllllll}
0 & 0 & 0 & 0 & 0 & 0 & 0 & 0 & 0 & 0 & 0 & 0 & 0 & 0 & 1 & 1
\end{tabular} & \\
\hline \begin{tabular}{llllllll|lllllll}
0 & 0 & 0 & 0 & 0 & 0 & 0 & 0 & 0 & 0 & 0 & 0 & 0 & 0 & 0
\end{tabular} & 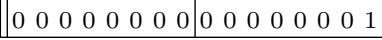 & \\
\hline \begin{tabular}{llllllll|llllllll}
0 & 0 & 0 & 0 & 0 & 0 & 0 & 0 & 0 & 0 & 0 & 0 & 0 & 0 & 0 & 0
\end{tabular} & 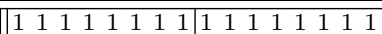 & \\
\hline \begin{tabular}{llllllll|llllllll}
0 & 0 & 0 & 0 & 0 & 0 & 0 & 0 & 0 & 0 & 0 & 0 & 0 & 0 & 0 & 0
\end{tabular} & $\left|\begin{array}{llllllll|l}0 & 1 & 0 & 1 & 0 & 1 & 0 & 1\end{array}\right|$ & \\
\hline \begin{tabular}{llllllll|llllllll}
0 & 0 & 0 & 0 & 0 & 0 & 0 & 0 & 0 & 0 & 0 & 0 & 0 & 0 & 0 & 0
\end{tabular} & $\mid$\begin{tabular}{llllllll|lllllll}
0 & 0 & 1 & 1 & 0 & 0 & 1 & 1 & 0 & 0 & 1 & 1 & 0 & 0 & 1
\end{tabular} & \\
\hline \begin{tabular}{llllllll|llllllll}
0 & 0 & 0 & 0 & 0 & 0 & 0 & 0 & 0 & 0 & 0 & 0 & 0 & 0 & 0 & 0
\end{tabular} & $\mid$\begin{tabular}{llllllll|llllllll}
0 & 0 & 0 & 1 & 0 & 0 & 0 & 1 & 0 & 0 & 0 & 1 & 0 & 0 & 0 & 1
\end{tabular} & \\
\hline \begin{tabular}{llllllll|llllllll}
0 & 0 & 0 & 0 & 0 & 0 & 0 & 0 & 0 & 0 & 0 & 0 & 0 & 0 & 0 & 0
\end{tabular} & $\mid$\begin{tabular}{llllllll|llllllll}
0 & 0 & 0 & 0 & 1 & 1 & 1 & 1 & 0 & 0 & 0 & 0 & 1 & 1 & 1 & 1
\end{tabular} & \\
\hline 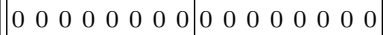 & $\left.\mid \begin{array}{llllllll|llllllll}0 & 0 & 0 & 0 & 0 & 1 & 0 & 1 & 0 & 0 & 0 & 0 & 0 & 1 & 0 & 1\end{array}\right]$ & \\
\hline $\left.\begin{array}{llllllll|llllllll}0 & 0 & 0 & 0 & 0 & 0 & 0 & 0 & 0 & 0 & 0 & 0 & 0 & 0 & 0 & 0\end{array}\right]$ & $\mid$\begin{tabular}{llllllll|llllllll}
0 & 0 & 0 & 0 & 0 & 0 & 1 & 1 & 0 & 0 & 0 & 0 & 0 & 0 & 1 & 1
\end{tabular} & \\
\hline \begin{tabular}{llllllll|llllllll}
0 & 0 & 0 & 0 & 0 & 0 & 0 & 0 & 0 & 0 & 0 & 0 & 0 & 0 & 0 & 0
\end{tabular} & \begin{tabular}{llllllll|llllllll}
0 & 0 & 0 & 0 & 0 & 0 & 0 & 1 & 0 & 0 & 0 & 0 & 0 & 0 & 1
\end{tabular} & \\
\hline \begin{tabular}{|llllllll|lllllll} 
& 0 & 0 & 0 & 0 & 0 & 0 & 0 & 0 & 0 & 0 & 0 & 0 & 0 & 0
\end{tabular} & 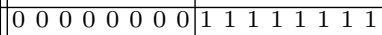 & \\
\hline \begin{tabular}{llllllll|llllllll}
0 & 0 & 0 & 0 & 0 & 0 & 0 & 0 & 0 & 0 & 0 & 0 & 0 & 0 & 0 & 0
\end{tabular} & \begin{tabular}{llllllll|llllllll}
0 & 0 & 0 & 0 & 0 & 0 & 0 & 0 & 0 & 1 & 0 & 1 & 0 & 1 & 0 & 1
\end{tabular} & \\
\hline \begin{tabular}{lllllll|llllllll}
0 & 0 & 0 & 0 & 0 & 0 & 0 & 0 & 0 & 0 & 0 & 0 & 0 & 0
\end{tabular} & $\mid$\begin{tabular}{llllllll|llllllll}
0 & 0 & 0 & 0 & 0 & 0 & 0 & 0 & 0 & 0 & 1 & 1 & 0 & 0 & 1 & 1
\end{tabular} & \\
\hline 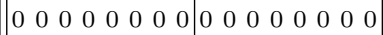 & $\mid \begin{array}{llllllllllllll}0 & 0 & 0 & 0 & 0 & 0 & 0 & 0 & 0 & 0 & 0 & 1 & 0 & 0\end{array}$ & \\
\hline \begin{tabular}{llllllll|llllllll}
0 & 0 & 0 & 0 & 0 & 0 & 0 & 0 & 0 & 0 & 0 & 0 & 0 & 0 & 0 & 0
\end{tabular} & $\left.\begin{array}{llllllll|llllllll}0 & 0 & 0 & 0 & 0 & 0 & 0 & 0 & 0 & 0 & 0 & 0 & 1 & 1 & 1 & 1\end{array}\right]$ & \\
\hline \begin{tabular}{llllllll|llllllll}
0 & 0 & 0 & 0 & 0 & 0 & 0 & 0 & 0 & 0 & 0 & 0 & 0 & 0 & 0 & 0
\end{tabular} & $\mid$\begin{tabular}{llllllll|llllllll}
0 & 0 & 0 & 0 & 0 & 0 & 0 & 0 & 0 & 0 & 0 & 0 & 0 & 1 & 0 & 1
\end{tabular} & \\
\hline \begin{tabular}{llllllll|llllllll}
0 & 0 & 0 & 0 & 0 & 0 & 0 & 0 & 0 & 0 & 0 & 0 & 0 & 0 & 0 & 0
\end{tabular} & \begin{tabular}{llllllll|llllllll}
0 & 0 & 0 & 0 & 0 & 0 & 0 & 0 & 0 & 0 & 0 & 0 & 0 & 0 & 1 & 1
\end{tabular} & \\
\hline $\begin{array}{llllllll}0 & 0 & 0 & 0 & 0 & 0 & 0 & 0\end{array}$ & & \\
\hline
\end{tabular}

algorithm exploits the fractal structure observed inside the binomial sequences. In Table 3, the 32 first binomial sequences are depicted. It can be noticed that, except for the rightmost column, the rest of the table can be considered as a $(32 \times 32)$ matrix divided into four $(16 \times 16)$ matrices $\left(\frac{A \| B}{\bar{C}|| D}\right)$, where the matrices $A, B$ and $D$ are equal while $C$ is the identically null matrix. Inside the $(16 \times 16)$ matrices $A, B$ and $D$ the previous pattern is found again. The same happens inside the corresponding $(8 \times 8)$ sub-matrices of $A, B$ and $D$, and so on. The key idea of this algorithm consists in:

1. Associating each binary sequence with period $T=2^{m}$ with a $\left(2^{m} \times 2^{m}\right)$ matrix $M$ corresponding to the first $2^{m}$ binomial sequences.

2. Reducing in $\log T=m$ steps the size of the matrix $M$ until the final binomial sequence $\left\{\left(\begin{array}{l}n \\ i_{t}\end{array}\right)\right\}$ is selected. Then, according to item 4 in Section 3, the linear complexity of the sequence is $L C=i_{t}+1$.

Consider the sequence $\mathbf{s}=\left\{s_{n}\right\}(n=0,1,2, \ldots, T-1)$ with period $T=2^{m}$. Then, s can be decomposed into two sub-sequences of length $T / 2$, that is:

$$
L(\mathbf{s})=\left\{s_{0}, s_{1}, \ldots s_{T / 2-1}\right\} \text { and } R(\mathbf{s})=\left\{s_{T / 2}, s_{T / 2+1}, \ldots s_{T-1}\right\},
$$


where $\mathbf{s}=(L(\mathbf{s}), R(\mathbf{s}))$. According to the binomial representation of $\mathbf{s}$, if the bitwise XOR between both sub-sequences is performed, then the binomial sequences in the matrices $A$ and $B$ are cancelled and a new sequence $\mathbf{r}=L(\mathbf{s}) \oplus R(\mathbf{s})$ with period $k=2^{m-1}$ and linear complexity $L C \in[T / 2+1, \ldots, T]$ is obtained. Repeating the same procedure, we will get a new sequence $\mathbf{r}$ with period $k=$ $2^{m-2}$ and linear complexity $L C \in[3 T / 4+1, \ldots, T]$. If, at a particular step, the sequence $\mathbf{r}$ happens to be the identically null sequence, that is $(L(\mathbf{s})=R(\mathbf{s}))$, then the algorithm just takes half the sequence, $\mathbf{s} \longleftarrow R(\mathbf{s})$, and the computation goes on. After $m$ steps we get the final linear complexity of the original sequence. The algorithm is summarized as follows:

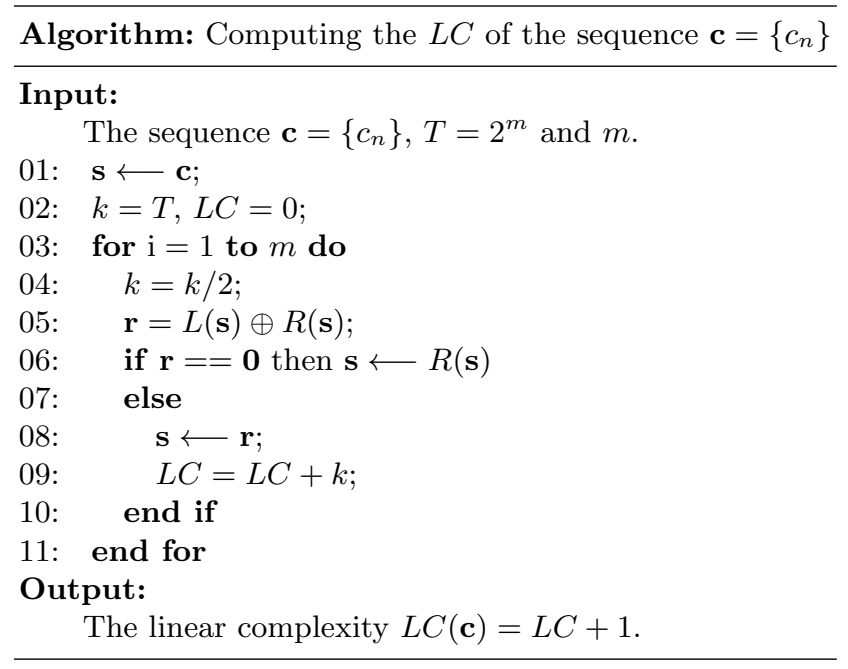

Recall that the algorithm above described just involves the Exclusive-OR logic operation, so that the computation of $L C$ is reduced to a bit-wise addition between the two halves of a binary sequence. Consequently, the algorithm efficiency is quite evident. In brief, we provide the value of the linear complexity of a binary sequence in $m$ steps at the price of minimal computational operations. Compared with the Berlekamp-Massey algorithm, the proposed algorithm needs half the sequence required by that algorithm cited in [11].

\subsection{Application of the algorithm to the generalized sequences}

The previous algorithm requires the knowledge of the entire sequence $\mathbf{c}$ to compute its $L C$. Nevertheless, when the algorithm is applied to sequences with an upper-bound on their $L C$, e.g. the generalized sequences, then a modification can be introduced to reduce the amount of processed bits. The main idea is to stop the algorithm at any step $m_{0}<m$, in which the form of the resulting sequence $\mathbf{r}$ allows us to compute the $L C$ with a minimum number of known bits. Let us see a simple example. 
Let $\mathbf{c}=(1,1,1,0,0,0,1,0,0,1,1,1,0,1,0,0)$ be a generalized sequence of period $T=2^{4}$ obtained from a LFSR with $L=5$, connection polynomial $p(x)=$ $x^{5}+x^{2}+1$ and shift $p=2$. In Table 3 , the $(16 \times 16)$ matrix $A$ tells us that $\mathbf{c}$ is a linear combination of the binomial sequences $\left\{\left(\begin{array}{c}n \\ 0\end{array}\right)\right\}-\left\{\left(\begin{array}{c}n \\ 12\end{array}\right)\right\}$, where $\left\{\left(\begin{array}{c}n \\ 12\end{array}\right)\right\}$ corresponds to the upper-bound on the $L C$ given in equation (1).

At step $i=1$ of the algorithm, we have that:

$$
\mathbf{r}=(1,1,1,0,0,0,1,0) \oplus(0,1,1,1,0,1,0,0)=(1,0,0,1,0,1,1,0)
$$

and the binomial sequences $\left\{\left(\begin{array}{l}n \\ 0\end{array}\right)\right\}-\left\{\left(\begin{array}{l}n \\ 7\end{array}\right)\right\}$ of the $(8 \times 8)$ sub-matrices $A$ and $B$ have been cancelled. Moreover, the resulting sequence $\mathbf{r}$ has period $k=8$ and it is just the linear combination of the binomial sequences $\left\{\left(\begin{array}{l}n \\ 8\end{array}\right)\right\}-\left\{\left(\begin{array}{c}n \\ 12\end{array}\right)\right\}$, see the $(8 \times 8)$ sub-matrix $D$. It is easy to see that if the components of $\mathbf{r}$ satisfy jointly

$$
r_{0}=\bar{r}_{4}, r_{1}=\bar{r}_{5}, r_{2}=\bar{r}_{6}, r_{3}=\bar{r}_{7},
$$

then $\left\{\left(\begin{array}{c}n \\ 12\end{array}\right)\right\}=\{0,0,0,0,1,1,1,1\}$ has to be included in the binomial representation. Thus, $i_{t}=12$ and $L C=i_{t}+1=13$. In order to check if $L(\mathbf{r})=\bar{R}(\mathbf{r})$, it is enough to compare two bits of $\mathbf{r}$, that is to check whether

$$
r_{0} \neq r_{4}
$$

At the same time, recall that $r_{0}=c_{0} \oplus c_{8}$ and $r_{4}=c_{4} \oplus c_{12}$. Therefore, the knowledge of only 4 bits $\left(c_{0}, c_{4}, c_{8}, c_{12}\right)$ of the sequence $\mathbf{c}$ (a quarter of the entire sequence, $T / 4$ ) and the previous condition allow us to compute, at step $i=1$, the linear complexity of $\mathbf{c}$, here $L C(\mathbf{c})=13$.

The same improvement applies for other generalized sequences with $L$ satisfying:

$$
L-2=2^{i}-1 \quad i>1 \text {, }
$$

that is for $L=5,9,17,33, \ldots$ stop the algorithm when the period of the sequence r equals $2^{3}, 2^{4}, 2^{5}, 2^{6}, \ldots$, respectively. Under these conditions, their corresponding complexities $L C$ can be computed with the knowledge of $T / 4, T / 8, T / 16$, $T / 32, \ldots$ bits of the corresponding generalized sequence, respectively.

\section{Conclusion}

In this work, an efficient algorithm to compute the linear complexity of binary sequences with period $T=2^{m}$ has been introduced and developed. In fact, the proposed algorithm reduces the linear complexity computation to $m$ bit-wise XOR logic operations among the bits of the sequence. The amount of sequence needed for this computation is half the amount needed by the well-known BerlekampMassey algorithm. The core of the proposed algorithm is the fractal structure of the binomial sequences in which the analysed sequence is decomposed. Although the algorithm is general, its application to a family of cryptographic sequences (the generalized sequences) improves the efficient of this procedure as it reduces the amount of sequence to be processed. The extension of this algorithm to other families of cryptographic sequences and its consequent improvement is proposed as future work. 


\section{References}

1. Cardell, S. D., Fúster-Sabater, A.: Linear models for the self-shrinking generator based on CA. Journal of Cellular Automata 11(2-3), 195-211 (2016)

2. Cardell, S. D., Fúster-Sabater, A.: Discrete linear models for the generalized selfshrunken sequences. Finite Fields and Their Applications 47, 222-241 (2017)

3. Cardell, S. D., Fúster-Sabater, A.: The t-Modified Self-shrinking Generator. In: Shi, Y. et al. (eds.): ICCS 2018, LNCS, vol. 10860, pp. 653-663, Springer, Heidelberg (2018). https://doi.org/10.1007/978-3-319-93698-7_50

4. Cardell, S. D., Fúster-Sabater, A.: Binomial representation of cryptographic binary sequences and its relation to cellular automata. Complexity, 2019, Article ID 2108014, 1-13 (2019).

5. Coppersmith, D., Krawczyk, H., Mansour, Y.: The Shrinking Generator. In: Stinson, Douglas R. (eds.) CRYPTO'93, LNCS, vol. 773, pp. 22-39. Springer, Heidelberg (1994)

6. Díaz Cardell, S., Fúster-Sabater, A.: Cryptography with Shrinking Generators: Fundamentals and Applications of Keystream Sequence Generators Based on Irregular Decimation. Springer Briefs in Mathematics. Springer International Publishing, Switzerland (2019)

7. Fúster-Sabater, A.: Generation of Cryptographic Sequences by means of Difference Equations. Applied Mathematics \& Information Sciences 8(2), 475-484 (2014)

8. Fúster-Sabater, A., Cardell, S. D.: Linear complexity of generalized sequences by comparison of PN-sequences. RACSAM, 114(2), 79-97 (2020). https://doi.org/10.1007/s13398-020-00807-5

9. Golomb, S.W.: Shift Register-Sequences. Aegean Park Press, Laguna Hill, California (1982)

10. Hu, Y., Xiao, G.: Generalized self-shrinking generator. IEEE Transactions on Information Theory 50(4), 714-719 (2004)

11. Massey, J.L.: Shift-register synthesis and BCH decoding. IEEE Transactions on Information Theory 15(1), 122-127 (1969)

12. Meier, W., Staffelbach, O.: The self-shrinking generator. In: Cachin, C., Camenisch, J. (eds.) Advances in Cryptology - EUROCRYPT 1994, LNCS, vol. 950, pp. 205214. Springer, Heidelberg (1994).

13. United States Patent. Inventors: Chang, K. Y. (Daejeon, KR) et al. Electronics and Telecommunications Research Institute (Daejeon, KR). Document Identification: US 20060098820 A1. Date: May 11, 2006.

14. Wolfram, S.: Cellular automata as simple self-organizing system. In: Caltrech preprint CALT, pp. 68-938. Calif Inst. of Technology, Pasadena, CA (1982) 